\title{
EMW Distribution for Human Hormone
}

\author{
Geetha. $\mathrm{T}^{*}$ and Poongothai. $\mathrm{T}^{* *}$ \\ * Asst. Professor of Mathematics, K.N. Govt. Arts College, Thanjavur, Tamilnadu, India \\ ** Research Scholar, K.N. Govt, Arts College, Thanjavur.
}

\begin{abstract}
A stochastic modeling of biological systems is crucial to effectively and efficiently developing treatments for medical conditions that plague humanity. The study of challenge tests designed to evaluate serotoninergic pathways have widely used intravenous citalopram. Oral citalopram has also been used, but unsatisfactory results were obtained with a dose of $20 \mathrm{mg}$. We evaluated cortisol, growth hormone and prolactin levels and determine whether a higher oral dose would reproduce similar to those described for intravenous administration. Under the assumption that the threshold level of cortisol is a random variable follows exponentiated modified weibull distribution. The survival function of cortisol and its p.d.f are derived.
\end{abstract}

Keywords: Cortisol, threshold, EMWD, cumulative Damage process.

\section{INTRODUCTION}

K.Chandrasekar and R.Kannan has been derived the stochastic model for estimating the expected time to seroconversion of HIV infected using exponentiated modified modified weibull distribution. In this paper, the stochastic model for the expected time to cortisol and variance of the cortisol time are derived under the assumption that threshold level of stress effect is a random variable which follows exponentiated modified weibull distribution.

Challenge tests to evaluate serotoninergic function in psychiatric disorders are common and usually evaluate corticotrophin-releasing hormone, growth hormone and prolactin. Fenfluramine has given way to other agents, including 5-HT reuptake inhibitors (SSRI antidepressants). Although paroxetine has been used in challenge tests, citalopram is often the SSRI of choice due to its higher selectivity. In the only double-blind randomized study addressing 5-HT-mediated neuroendocrine response, no difference was found between citalopram, a racemic compound, and escitalopram, its active isomer. Pharmacokinetic studies have shown that maximum plasma levels of approximately $130-160 \mathrm{nmol} / \mathrm{l}$ are generally obtained within $2-4 \mathrm{~h}$ after a $50-\mathrm{mg}$ oral dose. A second, lower, peak is sometimes observed. The decline is considered to be monoexponential with a $t_{1 / \text { of approximately } 30 \mathrm{~h} \text {. Although intravenous }}$ citalopram has previously been used in serotoninergic challenge tests, this formulation is not available in many countries, and the only challenge test with oral citalopram (at a dose of 20 $\mathrm{mg}$ ) produced less than satisfactory results. The objectives of this study were: 1) to measure plasma levels of cortisol, growth hormone and prolactin as a means of evaluating the neuroendocrine response to a higher dose $(40 \mathrm{mg}$ ) of oral citalopram used as a tool to stimulate hormone secretion in neuroendocrine challenge paradigms; 2) to compare the kinetics of serum citalopram levels and neuroendocrine response using this higher dose of citalopram.
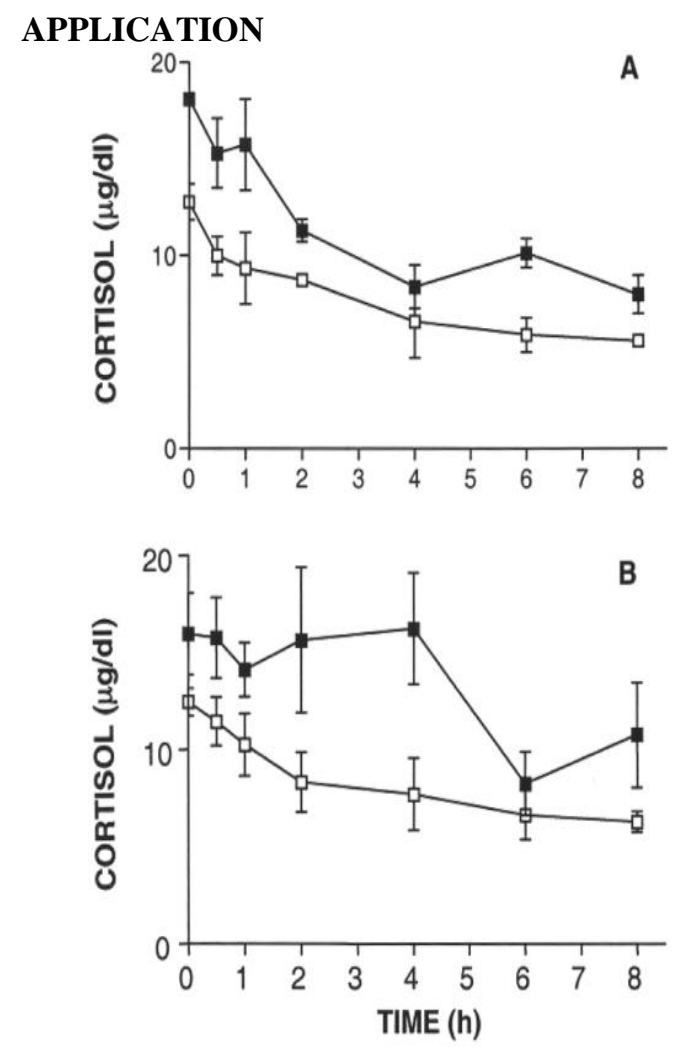

Figure 1 - Plasma levels of cortisol. Mean cortisol levels (+SEM) of volunteers 4 and 7 (A) and volunteers $5,6,8$ and 9 (B) after placebo

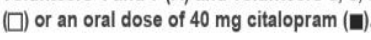


Growth hormone levels increased in three of the four subjects who presented a rise I cortisol level and a peak at 2-3 h after citalopram intake. Curiously, the three subjects who did not present an increase in growth hormone levels after citalopram intake actually a peak at 2-3 h after placebo intake. The reason for this is unknown. In two of the six subjects, prolactin levels increased after citalopram challenge. All measurements were within the normal range, except for that of one subject who had a baseline prolactin level above the upper normal limit, which might have been related to stress or even to a normally occurring pulse outside normal limits.

\section{MATHEMATICAL MODEL}

$X_{\mathrm{i}}$ : time for cortisol level during exercise.

Y : Cortisol level.

$U_{i}$ : a continuous random variable denoting the inter arrival times between successive contacts with p.d.f $f($.$) and c.d.f F($.$) .$

$g_{k}($.$) : the p.d.f of random variable.$

$\mathrm{T}$ : a continuous random variable denoting the time of cortisol with p.d.f l(.) and c.d.f L(.).

$V_{i}(\mathrm{t})$ : probability of exactly i contacts in $(0, \mathrm{t}]$

$I^{*}(\mathrm{~s})$ : is the Laplace stieltje's transform of $1(\mathrm{t})$. $f^{*}(\mathrm{~s})$ : is the Laplace stieltje's transform of $\mathrm{f}(\mathrm{t})$.

The probability density function of exponentiated modified weibull distribution is,

$$
\begin{aligned}
& \mathrm{h}(\mathrm{x}) \quad=\quad \alpha \quad\left(\theta_{+} \quad \gamma \beta x^{\beta-1}\right) \\
& e^{-\left(\theta x+\gamma x^{\beta}\right)}\left[1-e^{-\left(\theta x+\gamma x^{\beta}\right)}\right]^{\alpha-1}
\end{aligned}
$$

$\mathrm{x}>0$ and $\theta_{s} \beta_{s} \alpha_{s} \gamma>0$

and its distribution function is

$\mathrm{H}(\mathrm{x})=\left[1-e^{-\left(\theta x+y x^{9}\right)}\right]^{\alpha}, \mathrm{x}>0$

then its survival function is

$\bar{H}(\mathrm{x})=1-\left[1-e^{\left(\theta x+\gamma x^{\theta}\right)}\right]^{\alpha}$

Put $\alpha=2, \beta=1$ in $\bar{H}(\mathrm{x})$, then it becomes

$\bar{H}(\mathrm{x})=\left[2 e^{-x[\theta+\gamma)}-e^{-2 x(\theta+\gamma)}\right]$

since $f($.$) follows \exp (\mathrm{c})$, then

If $f^{*}(\mathrm{~s})=\frac{c}{c+\varepsilon}$ and

$l^{*}(\mathrm{~s})=\frac{2\left[1-g^{+}(\theta+Y)\right] \frac{c}{c+x}}{\left.\left[1-g^{*}(\theta+Y)\right] \frac{c}{c+s}\right]}-\frac{\left[1-g^{*} 2(\theta+Y)\right] \frac{c}{c+x}}{\left.\left[1-g^{+} 2(\theta+Y)\right] \frac{c}{c+s}\right]}$

where

$g^{*}(\theta+\gamma)=\frac{\mu}{\mu+(\theta+\gamma)}, g^{*}(2(\theta+\gamma))=\frac{\mu}{\mu+2(\theta+\gamma)}$

$\mathrm{E}(\mathrm{T})=\frac{a \mu+2(\theta+\gamma)}{2 \mathrm{c}(\theta+7)}$

$\mathrm{V}(\mathrm{T})=\frac{5 \mu^{2}+4\left(\theta^{2}+\eta^{2}\right)+12 \mu(\theta+\eta)+8 \theta Y}{4 c^{2}(\theta+\gamma)^{2}}$

GRAPH: 1

\begin{tabular}{|c|c|c|c|c|c|c|}
\hline $\mathbf{X}$ & 0.6 & 1.2 & 2.4 & 4.7 & 7.1 & 9.4 \\
\hline $\mathbf{E}(\mathbf{T})$ & 1.7494 & 0.8748 & 0.4374 & 0.2233 & 0.1478 & 0.1117 \\
\hline $\mathbf{V}(\mathbf{T})$ & 3.0568 & 0.7642 & 0.1910 & 0.0498 & 0.0218 & 0.0125 \\
\hline
\end{tabular}

GRAPH: 2

\begin{tabular}{|c|c|c|c|c|c|c|}
\hline $\mathbf{X}$ & 0.6 & 1.2 & 2.4 & 4.7 & 7.1 & 9.4 \\
\hline $\mathbf{E}(\mathbf{T})$ & 1.7272 & 0.8636 & 0.4319 & 0.2205 & 0.1459 & 0.1102 \\
\hline $\mathbf{V ( T )}$ & 2.9821 & 0.7455 & 0.1864 & 0.0486 & 0.0213 & 0.0121 \\
\hline
\end{tabular}

GRAPH: 1

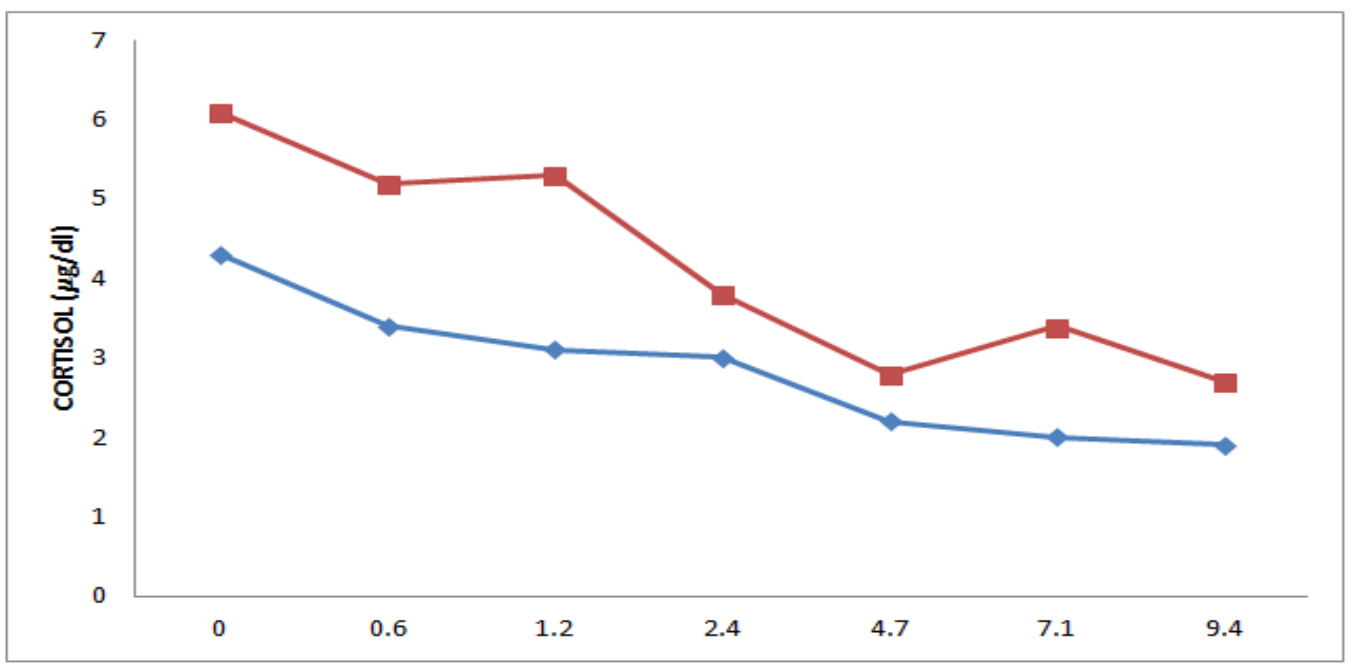

TIME (h) 
GRAPH: 2

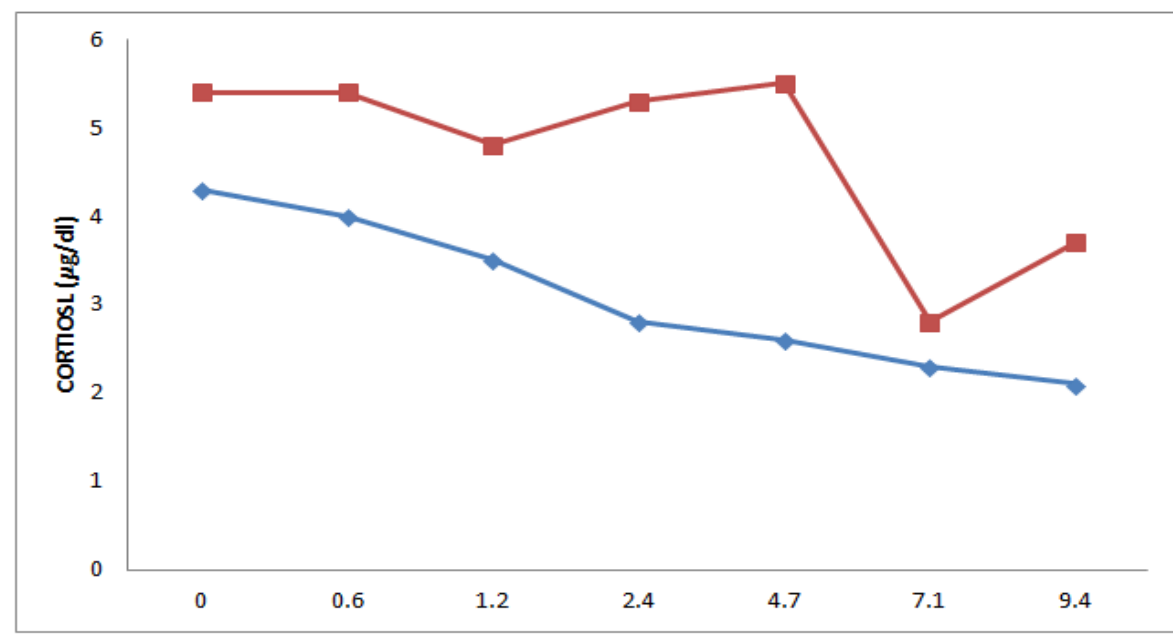

TIME (h)

GRAPH : 1

\begin{tabular}{|c|c|c|c|c|c|c|c|}
\hline $\mathbf{X}$ & 0 & 0.6 & 1.2 & 2.4 & 4.7 & 7.1 & 9.4 \\
\hline $\mathbf{Y 1}$ & 4.3 & 3.4 & 3.1 & 3 & 2.2 & 2 & 1.9 \\
\hline $\mathbf{Y 2}$ & 6.1 & 5.2 & 5.3 & 3.8 & 2.8 & 3.4 & 2.7 \\
\hline
\end{tabular}

GRAPH: 2

\begin{tabular}{|c|c|c|c|c|c|c|c|}
\hline $\mathbf{X}$ & 0 & 0.6 & 1.2 & 2.4 & 4.7 & 7.1 & 9.4 \\
\hline $\mathbf{Y 1}$ & 4.3 & 4 & 3.5 & 2.8 & 2.6 & 2.3 & 2.1 \\
\hline $\mathbf{Y 2}$ & 5.4 & 5.4 & 4.8 & 5.3 & 5.5 & 2.8 & 3.7 \\
\hline
\end{tabular}

\section{CONCLUSION}

The threshold level of cortisol is a random variable follows exponentiated modified weibull distribution and the survival function of cortisol and its p.d.f. also found.

\section{REFERENCES}

[1]. Attenburrow MJ, Mitter PR, Whale R, Terao T, Cowen PJ. Low-dose Citalpram as a 5HT neuroendocrine probe. Psychopharmacology (Berl). 2001; 155(3) : 323-6.

[2]. Chandrasekar. K and Kannan.R (2013), A Stochastic Model for Estimation of Expected time to Seroconversion of HIV infected using exponential-geometric distribution, Bio-Science Research Bulletin, Vol. 29(1), 29-38.

[3]. Elbatal.L., (2011), Exponentaited modified Weibull distribution, Economic Quality Control, 26, 189-200.

[4]. Esary, J.D., Marshall, A.W. and Proschan, F. (1973), Shock models and Wear processes, Ann. Probability, 1(4) : 627-649.

[5]. Hanley NR, Van de Kar LD. Serotonin and the neuroendocrine regulation of the hypothalamic - pituitary adrenal axis in health and diseases. Vitam Horm. 2003; 66: 189-255.

[6]. Kannan. R., Kavitha. S., and Sathiyamoorthi .R., (2011), A Stochastic Model for the Estimation of Time to Seroconversion of HIV Transmission using Exponentiated Exponential Distribution , Bio-Science Research Bulletin, Vol.27(2): 67-76.

[7]. Kannan. R., Venkatachalam. K. A., Sathiyamoorthi. R. and Malarvizi. G., (2007), A Stochastic Approach to Determine the Expected Time to Seroconversion of HIV infected. Journal of Indian Acad. of Math., 29(1), 237-249.

[8]. Sathiyamoorthi, R. and Kannan, R., (2001), A Stochastic Model for Time to Seroconversion of HIV Transmission, Journal of Kerala Statistical Association, Vol. 12: 23-28. 